\title{
Assessment of the Non-Cystic Fibrosis Bronchiectasis Severity: The FACED Score vs the Bronchiectasis Severity Index
}

\author{
J. Minov $^{*}, 1$, J. Karadzinska-Bislimovska ${ }^{1}$, K. Vasilevska ${ }^{2}$, S. Stoleski ${ }^{1}$ and \\ D. Mijakoski ${ }^{1}$
}

\author{
${ }^{I}$ Institute for Occupational Health of R. Macedonia - WHO Collaborating Center and GA ${ }^{2} L E N$ Collaborating Center, \\ Skopje, R. Macedonia \\ ${ }^{2}$ Institute for Epidemiology and Biostatistics, Skopje, R. Macedonia
}

\begin{abstract}
Introduction: Non-cystic fibrosis bronchiectasis (NCFB) is a multidimensional disease, and no single isolated parameter is proved to have sufficient power for any overall determination of its severity and prognosis.

Objective: To compare the results of the assessment of the NCFB severity with respect to its prognosis in the same patients by two different validated scores, i.e. the FACED score and the Bronchiectasis Severity Index (BSI).

Methods: An observational study including 37 patients with NCFB (16 males and 21 female aged 46 to 76 years) was performed. All patients underwent evaluation of the variables incorporated in the FACED score (FEV $\%$ predicted, age, chronic colonization by Pseudomaonas aeruginosa, radiological extent of the disease, and dyspnea) and in the BSI (age, body mass index, $\mathrm{FEV}_{1} \%$ predicted, hospitalization and exacerbations in previous year, dyspnea, chronic colonization by Pseudomaonas aeruginosa and other microrganisms, and radiological extent of the disease).
\end{abstract}

Results: According to the value of the derived overall FACED score we found 17 patients $(45.9 \%)$ with mild bronchiectasis, 14 patients $(37.8 \%)$ with moderate bronchiectasis and 6 patients $(16.2 \%)$ with severe bronchiectasis. The mean derived FACED score was $3.4 \pm 1.3$. In addition, according to the value of the derived overall BSI score, the frequency of patients with low, intermediate and high BSI score was 16 patients $(43,2 \%), 14$ patients $(37.8 \%)$ and 7 patients (18.9\%), respectively. The mean derived BSI score was $6.4 \pm 2.5$.

Conclusion: We found similar results by the assessment of the NCFB severity in regard to its prognosis by both the FACED score and the BSI. Further studies determining how these scores may impact clinical practice are needed.

Keywords: Assessment, bronchiectasis, prognosis, score, severity.

\section{INTRODUCTION}

Bronchiectasis is considered as a heterogeneous condition characterized by irreversible airway dilatation with chronic bronchial infection/inflammation. This is pathological description of a disease process that has many possible causes $[1,2]$.

Bronchiectasis is generally classified into cystic fibrosis and non-cystic fibrosis bronchiectasis (NCFB). NCFB may result from a number of congenital and acquired causes, with the later more frequent. Congenital causes include primary ciliary dyskinesia, primary immunodeficiencies, situs inversus, etc. Tuberculosis, pneumonia, inhaled foreign bodies, pulmonary aspiration, corticosteroid-dependent asthma, allergic bronchopulmonal aspergillosis and bronchial tumours are the

*Address correspondence to this author at the Department of Cardiorespiratory Functional Diagnostics, Institute for Occupational Health of R. Macedonia - WHO Collaborating Center and GA ${ }^{2}$ LEN Collaborating Center, Skopje, R. Macedonia, II Makedonska Brigada 43, 1000 Skopje, R. Macedonia; Tel: + 38922639 637; Fax: + 38922621 428;

E-mail:minovj@hotmail.com major acquired causes of NCFB $[3,4]$. Infective causes associated with NCFB include infections caused by Staphylococcus aureus, Klebsiella, Bordetella pertusis, etc. Various immunological disorders, such as childhood acquired immune deficiency syndrome (AIDS), inflammatory bowel disease (especially ulcerative colitis) and rheumatoid arthritis, are also linked to the development of NCFB [5, 6]. In a significant number of cases the underlying etiology of NCFB remains unidentified and is subsequently referred to as idiopathic [7]. In addition, there is evidence that $50 \%$ of patients with chronic obstructive pulmonary disease (COPD) have co-existent bronchiectasis [8].

$\mathrm{NCBF}$ is a common condition. According to the actual estimation, there are at least 110,000 adults in the USA with this condition causing economic costs at 630 million dollars per year $[9,10]$. As it is recognized that its prevalence increases around the world, bronchiectasis became an important public health problem [6]. With respect to this, there was a need for a predictive tool for assessment of the disease severity in routine practice which would allow 
targeting of therapies to the patients most likely to benefit and improving their quality of life [11]. In the recent years, two multidimensional grading systems capable of classifying the severity of bronchiectasis according to its prognosis were designed: the FACED score and Bronchiectasis Severity Index (BSI). The FACED score (forced expiratory volume in 1 second $\left(\mathrm{FEV}_{1}\right) \%$ predicted $[\mathrm{F}]$, age $[\mathrm{A}]$, chronic colonization by Pseudomonas aeruginosa [C], extension of the disease by radiological assessment [E] and dyspnea [D]) [12] is a five-point score that predicts probability of all-cause mortality after 5 years of follow-up, whereas the BSI [13] is a seven-point score that identifies patients with NCFB at risk for future mortality, hospitalization and exacerbations.

The present study aims to compare the results of the assessment of $\mathrm{NCBH}$ severity in the same patients done by the FACED score and by the BSI.

\section{MATERIALS AND METHODOLOGY}

\section{Study Design and Setting}

An observational study, i.e. comparison between NCFB severity scores derived in the same patients by the FACED score and the BSI, was performed at the Institute for Occupational Health of R. Macedonia, Skopje - WHO Collaborating Center and $\mathrm{GA}^{2} \mathrm{LEN}$ Collaborating Center in the period May-November 2014.

\section{Patients}

We examined 37 patients ( 16 males and 21 females) aged 46 to 76 years with stable bronchiectasis. The diagnosis was established according to the actual recommendations, i.e. by high-resolution computed tomography (HRCT) of the chest in the subjects with clinical presentation consistent to bronchiectasis [14-16].

Inclusion criteria were: clinically stable patients with NCFB with no antibiotic use in the preceding 4 weeks.

Exclusion criteria were: active malignancy, cystic fibrosis (CF), active mycobacterial disease, human immunodeficiency virus (HIV) infection, pulmonary fibrosis with secondary traction bronchiectasis, and treatment with a long-term antibiotic therapy.

All participants were informed about the study and their written consent was obtained.

\section{The FACED Score}

The FACED score incorporates 5 dichotomised variables:

1. $\mathrm{FEV}_{1} \%$ predicted (cut-off $50 \%$, maximum value 2 points),

2. Age (cut-off 70 years, maximum value 2 points),

3. Presence of chronic colonization by Pseudomonas aeruginosa (dichotomic, maximum value 1 point),

4. Radiological extension (number of lobes affected, cut-off 2 lobes, maximum value 1 point)

5. Dyspnea (cut-off grade II on the Medical Research Council [MRC] scale, maximum value 1 point).
An overall score is derived as a sum of the scores for each variable and it may range from 0 to 7 points. By this score, the bronchiectasis is classified into 3 severity classes: mild bronchiectasis (overall score 0-2 points), moderate bronchiectasis (overall score 3-4 points) and severe bronchiectasis (overall score 5-7 points) (12).

\section{BSI}

The BSI incorporates 9 variables:

1. Age: less than 50 years (0 points); $50-69$ years (2 points), $70-79$ years ( 4 points), more than 80 years $(6$ points)

2. Body mass index (BMI): less than 18.5 (2 points), more than 18.5 ( 0 points)

3. $\mathrm{FEV}_{1} \%$ predicted: less than $80 \%$ (0 points), $50-80 \%$ (1 point), $30-49 \%$ ( 2 points), less than $30 \%$ (3 points)

4. Hospital admission in previous year: no (0 points), yes (5 points)

5. Exacerbations in previous year: $0-2$ ( 0 points $), 3$ or more ( 2 points)

6. MRC dyspnea score: 1-3 (0 points), 4 (2 points), 5 (3 points)

7. Pseudomonas aeruginosa colonization: no (0 point), yes (3 points)

8. Colonization with other microorganisms: no (0 point), yes (1 point)

9. Radiological severity (more than 3 lobes involved or cystic bronchiectasis): no ( 0 points), yes ( 1 point)

An overall score is derived as a sum of the scores for each variable and it may range from 0 to 26 points According to the overall score value, the patients with bronchiectasis are classified into 3 BSI classes: patients with low BSI score (overall score 0-4 points), patients with intermediate BSI score (overall score 5-8 points) and patients with high BSI score (overall score 9 or more points) [13].

\section{Patient Analysis}

At the time of clinical assessment all patients were clinically stable, with no antibiotic use in the preceding 4 weeks. Classification of smoking status was done according to the World Health Organization (WHO) guidelines on definitions of smoking status [17]. Pack-years smoked (one pack-year denotes one year of smoking 20 cigarettes per day) were calculated according to the actual recommendations [18].

The severity of dyspnea was graduated according to the MRC breathlessness scale into 5 grades: grade 1 (patient is not troubled by breathlessness except on strenuous exercise), grade 2 (getting short breath when hurrying on the level or walking up a slight hill), grade 3 (walking slower than most people on the level, stopping after a mile or so, or stopping after 15 minutes walking at own pace), grade 4 (stopping for breath after walking about 100 yds or after a few minutes on level ground) and grade 5 (being too breathless to leave the house or being breathless when undressing) [19]. 
All patients underwent spirometry including measures of forced vital capacity $(\mathrm{FVC})$ and $\mathrm{FEV}_{1}$ with recording the best result from three measurements the values of which were within $5 \%$ of each other. The results of spirometry were expressed as percentages of the predicted values according to the actual recommendations of European Repsiratory Society (ERS) and American Thoracic Society (ATS) [20, 21].

Bacteriological status of the patients was assessed on spontaneous early-morning sputum samples. Chronic colonization was considered by isolation of potentially pathogenic bacteria in sputum culture on at least two occasions in a period of 3 months [22] as the predominant pathogen underwent bacterial growth most frequently over this period of time. Patients who were unable to provide sputum samples (e.g. due to absence of productive cough) were classified as non-colonized.

Radiological evaluation, i.e. the extent of bronchiectasis, was made according to the number of pulmonary lobes affected (with the lingula considered as independent lobe) and the degree of bronchial dilatation (tubular, varicose or cystic). A small bronchiectasis visible only in a single pulmonary segment was not considered, as this can appear in a significant proportion of the healthy population $[15,23]$.

\section{Statistical Analysis}

Data for quantitative variables were expressed as mean value with standard deviation (SD), while the frequencies variables were expressed as absolute values and the percentage of the total.

\section{RESULTS}

Characteristics of the patients enrolled in the study are shown Table 1.

The values of the FACED score variables are presented on Table 2.

According to the value of the derived overall score we found 17 patients $(45.9 \%)$ with mild bronchiectasis, 14 patients $(37.8 \%)$ with moderate bronchiectasis and 6 patients $(16.2 \%)$ with severe bronchiectasis (Fig. 1). The mean derived FACED score was $3.4 \pm 1.3$.

The values of the BSI variables are presented on Table 3 .

According to the value of the derived BSI score we found 16 patients $(43.2 \%)$ with low BSI score, 14 patients $(37.8 \%)$ with intermediate BSI score and 7 patients $(18.9 \%)$ with high BSI score (Fig. 2). The mean derived BSI score was $6.4 \pm$ 2.5 .

\section{DISCUSSION}

NCFB is a chronic progressive condition resulting from infection and inflammation of the airway leading to destruction and remodeling of the bronchial wall $[6,24]$. The clinical manifestations of the disease include chronic and commonly purulent expectoration, multiple exacerbations and progressive dyspnea. All these events cause progressive decline in the lung function and impairment of the quality of
Table 1. Characteristics of the study participants.

\begin{tabular}{|c|c|}
\hline Characteristics & Patients $(\mathbf{n}=\mathbf{3 7})$ \\
\hline \hline M/F ratio & 0.8 \\
Mean age (yrs) & $63.4 \pm 8.1$ \\
BMI (kg.m $\left.{ }^{-2}\right)$ & $24.3 \pm 3.7$ \\
Smoking status & \\
Daily smokers & $14(37.8 \%)$ \\
Pack-years smoked & $12.8 \pm 5.7$ \\
Ex-smokers & $5(13.5 \%)$ \\
Dyspnea (MRC score) & $1.83 \pm 0.63$ \\
Sputum production & $29(78.4 \%)$ \\
& $73.8 \pm 11.4$ \\
Mean FVC value (\% pred.) & $57.6 \pm 8.7$ \\
Mean FEV value (\% pred.) & \\
Chronic colonisation & $3(8.1 \%)$ \\
Pseudomonas aeruginosa & $6(16.3 \%)$ \\
Other microorganisms & $2.25 \pm 0.78$ \\
Number of lobes affected & $2.12 \pm 0.54$ \\
& $1.14 \pm 0.37$ \\
Exacerbation in previous year & \\
Hospitalization in previous year & $8(21.6 \%)$ \\
Chronic treatment in previous year & $3(8.1 \%)$ \\
Systemic antibiotics & $6(16.3 \%)$ \\
Macrolides & \\
Oral corticosteroids & \\
\hline
\end{tabular}

Numerical data are expressed as a mean value with standard deviation; the frequencies as a number and percentage of patients with certain variable.

M: male; F: female; yrs: years; BMI: body mass index; kg: kilogram; m: meter; MRC: Medical Research Council; FVC: forced vital capacity; $\mathrm{FEV}_{1}$ : forced expiratory volume in 1 second; $\%$ pred.: $\%$ of predicted value.

Table 2. Values of the FACED score variables.

\begin{tabular}{|c|c|}
\hline Variable & Patients $(\mathbf{n}=\mathbf{3 7})$ \\
\hline \hline FEV $_{1} \%$ predicted & \\
$<50 \%$ & $9(24.3 \%)$ \\
$>50 \%$ & $28(75.7 \%)$ \\
Age (yrs) & \\
$>70$ & $7(18.9 \%)$ \\
$<70$ & $30(81.1 \%)$ \\
Chronic colonization by Pseudomonas aeruginosa & \\
Yes & $3(8.1 \%)$ \\
No & \\
Radiological extent (HRCT) & \\
$>2$ lobes affected & $16(43.9 \%)$ \\
$<2$ lobes affected & $21(56.8 \%)$ \\
MRC dyspnea score & \\
$>2$ (II) & $17(45.9 \%)$ \\
$<2$ (II) & $20(54.1 \%)$ \\
\hline
\end{tabular}

Frequencies are expressed as a number and percentage of patients with certain variable. $\mathrm{FEV}_{1}$ : forced expiratory volume in 1 second; yrs: years; HRCT: high-resolution computed tomography; MRC: Medical Research Council.

life of the patients $[25,26]$. As with other airway diseases (e.g. with COPD), the severity and prognosis of bronchiectasis can not sufficiently be assessed by one single parameter, so multidimensional approach to this issue is required. Most respiratory diseases have a disease-specific 


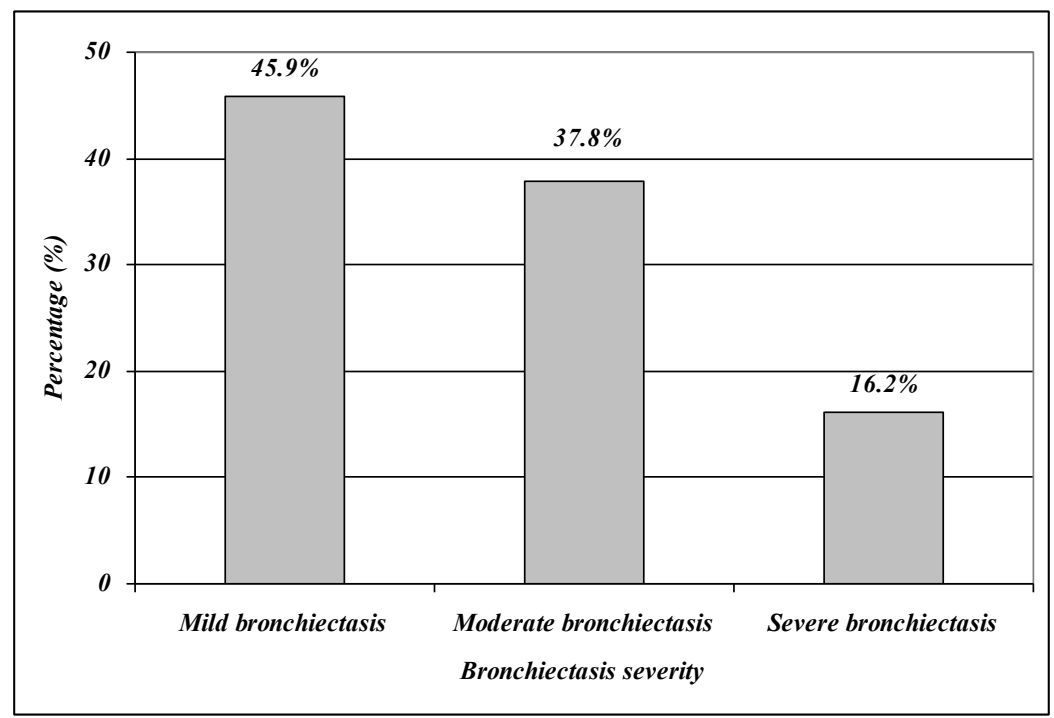

Fig. (1). Distribution of the patients by the FACED score.

Table 3. Values of the BSI variables.

\begin{tabular}{|c|c|}
\hline Variable & Patients $(n=37)$ \\
\hline $\begin{array}{l}\text { Age (yrs) } \\
<50 \\
50-69 \\
70-79 \\
>80\end{array}$ & $\begin{array}{c}8(21.6 \%) \\
22(59.4 \%) \\
7(18.9 \%) \\
/\end{array}$ \\
\hline $\begin{array}{l}\text { BMI }\left(\mathrm{kg} \cdot \mathrm{m}^{-2}\right) \\
\quad<18.5 \\
\quad>18.5\end{array}$ & $\begin{array}{c}2(5.4 \%) \\
35(94.6 \%)\end{array}$ \\
\hline $\begin{array}{c}\mathrm{FEV}_{1} \% \text { predicted } \\
>80 \% \\
50-80 \% \\
30-49 \% \\
<30 \%\end{array}$ & $\begin{array}{c}5(13.5 \%) \\
23(62.2 \%) \\
9(24.3 \%) \\
/ /\end{array}$ \\
\hline $\begin{array}{c}\text { Hospital admission in previous year } \\
\text { No } \\
\text { Yes }\end{array}$ & $\begin{array}{c}6(16.2 \%) \\
31(83.8 \%)\end{array}$ \\
\hline $\begin{array}{c}\text { Exacerbations in previous year } \\
\text { No } \\
\text { Yes }\end{array}$ & $\begin{array}{l}21(56.7 \%) \\
16(43.3 \%)\end{array}$ \\
\hline $\begin{array}{c}\text { MRC dyspnea score } \\
1-3 \\
4 \\
5\end{array}$ & $\begin{array}{c}33(89.2 \%) \\
4(10.8 \%) \\
/ /\end{array}$ \\
\hline $\begin{array}{c}\text { Pseudomonas aeruginosa colonization } \\
\text { No } \\
\text { Yes }\end{array}$ & $\begin{array}{c}34(91.9 \%) \\
3(8.1 \%)\end{array}$ \\
\hline $\begin{array}{c}\text { Colonization with other microorganisms } \\
\text { No } \\
\text { Yes }\end{array}$ & $\begin{array}{c}31(83.7 \%) \\
6(16.3 \%)\end{array}$ \\
\hline $\begin{array}{c}\text { Radiological extent (HRCT) } \\
>3 \text { lobes affected or cystic bronchiectasis } \\
\text { No } \\
\text { Yes }\end{array}$ & $\begin{array}{l}27(73.0 \%) \\
10(27.0 \%)\end{array}$ \\
\hline
\end{tabular}

Frequencies are expressed as a number and percentage of patients with certain variable. Yrs: years; BMI: body mass index; kg: kilogram; m: meter; $\mathrm{FEV}_{1}$ : forced expiratory volume in 1 second; MRC: Medical Research Council; HRCT: high-resolution computed tomography. severity assessment tool. The severity assessment is needed for appropriate treatment of the patients, stratification of the risk of complications, reduction of the costs associated with particular disease, as well as for researching activities (i.e. identification groups of patients likely to benefit from novel therapies) $[5,27,28]$.

Up to recent years there was no any scoring system for assessment of the NCFB severity and prognosis, and now there are two scoring systems designed for this purpose, the FACED score and the BSI. These scores have different structure and somewhat different aims. The FACED score is easy-to-use tool incorporating 5 dichotomic variables, whereas the BSI is relatively complex, awarding different point values for each of the variables and including multiple variables. In addition, the FACED score is aimed at prediction of the probability of all-cause mortality of the patients with NCFB in the next 5 years, whereas the BSI is aimed at identification of the patients with NCFB with higher risk for future mortality, hospitalization and exacerbations $[12,13]$.

In the present study we compared the results from the assessment of severity of the disease in patients with NCFB by these two scoring systems. The study group included patients with stable NCFB of both sexes aged 46 to 76 years. The study work-up consisted of evaluation of each variable incorporated in the FACED score and in the BSI. The scores derived by both scoring systems were similar. Namely, by the FACED score we found that the frequency of mild, moderate and severe bronchiectasis was approximately $46 \%$, $38 \%$ and $16 \%$, respectively. According to the score derived by the BSI, we found that the frequency of patients with low, intermediate and high BSI was $43 \%, 38 \%$ and $19 \%$, respectively. As in the available literature we did not find similar study, we can not compare the results obtained in our study with results of other studies. Taking into account the public health aspects of bronchiectasis, we expect that further studies for more precise determining the role of these 


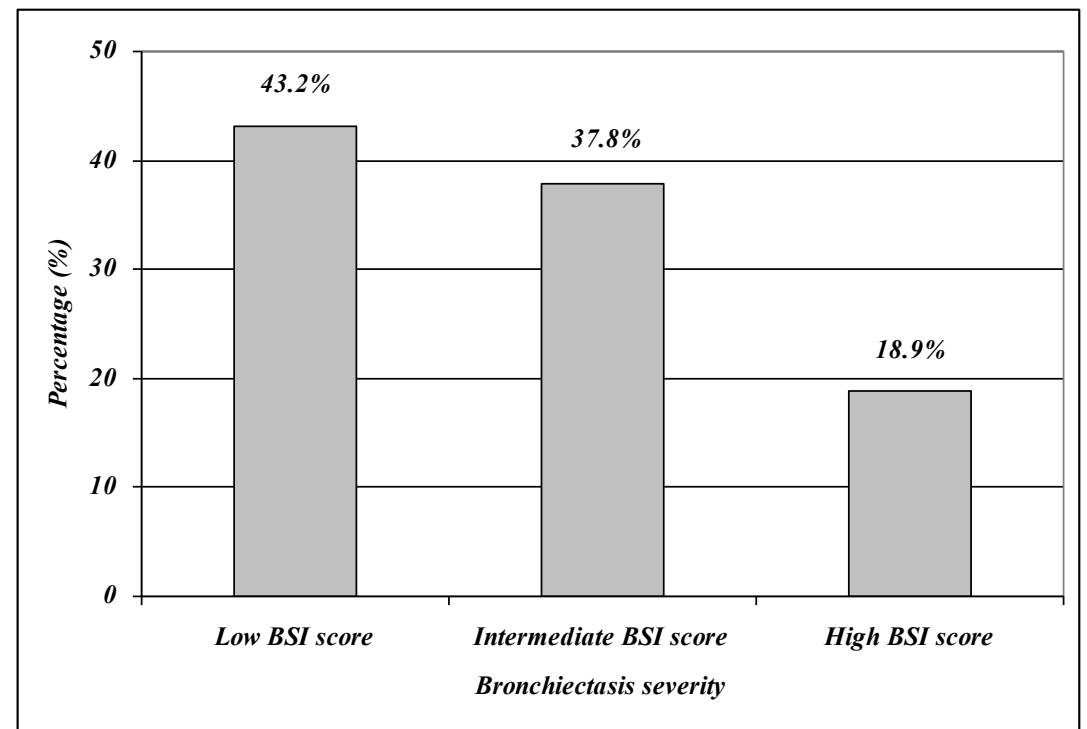

Fig. (2). Distribution of the patients by the BSI score.

scoring systems in the clinical practice and research area will be performed soon.

The present study has some limitations. Firstly, relatively small number of the subjects in the study group could have certain implications on the data obtained and its interpretation. Secondly, the majority of the patients in our study suffered from idiopathic and post-infective bronchiectasis, so the study is not powered to detect anything other than very large effects on survival in bronchiectasis due to less common etiologies (e.g. bronchiectasis in patients with systemic diseases). The strength of the study is the comparison of the results of two different predictive tools for assessment of the severity of the NCFB with respect to its prognosis.

In conclusion, in an observational study aimed at comparison of the results of the NCFB severity assessment with respect to its prognosis similar results were obtained by both the FACED score and the BSI. Our findings indicate that both scores can be used in routine clinical work, but there is a need for further studies in order to determine how these scores may impact the clinical practice.

\section{AUTHORS PARTICIPATIONS}

JM participated in the study design, writing the protocol, data collection, managing the analyses of the study, and writing all versions of the manuscript. JKB participated in the study design, writing the protocol, managing the analyses of the study, as well as writing all versions of the manuscript. KV participated in the study design and in the managing of the analyses of the study. SS and DM participated in the data collection and in the managing of the analyses of the study. All authors read and approved the final manuscript.

\section{CONFLICT OF INTEREST}

The authors confirm that this article content has no conflict of interest.

\section{ACKNOWLEDGEMENTS}

Declared none.

\section{REFERENCES}

[1] King P, Holdsworth S, Freezer N, Holmes P. Bronchiectasis. Int Med 2006; 36(11): 729-37.

[2] Neves PC, Guerra M, Ponce P, Miranda J, Vouga L. Non-cystic fibrosis bronchiectasis. Interact Cardiovasc Thorac Surg 2011; 13: 619-25.

[3] Hogg C. Primary ciliary dyskinesia: when to suspect the diagnosis and how to confirm it. Pediatr Respir Rev 2009; 10: 44-50.

[4] Lujan M, Gallardo X, Amengual MJ, Bosque M, Mirapeix RM, Domingo $\mathrm{CH}$. Prevalence of bronchiectasis in asthma according to oral steroid requirements: influence of immunoglobulin levels. Biomed Research International 2013. Article ID: 109219. http://dx.doi.org/10.1155/2013/109219

[5] Hill AT, Pasteur M, Cornford C, Welham S, Bilton D. Primary care summary of the British Thoracic Society Guideline on the management of non-cystic fibrosis bronchiectasis. Prim Care Respir J 2011; 20 (2): 135-40.

[6] O’Donnel AF. Bronchiectasis. Chest 2008; 134 (4): 815-23.

[7] Lazarus A, Myers J, Fuhrer G. Bronchiectasis in adults: a review. Postgrad Med 2008; 120(3): 113-21.

[8] Patel IS, Vlahos I, Wilkinson TM, et al. Bronchiectasis, exacerbation indices, and inflammation in chronic obstructive pulmonary disease. Am J Respir Crit Care Med 2004; 170: 400-7.

[9] Weycker D, Edelsberg J, Oster G, Tino G. Prevalence and economic burden of bronchiectasis. Clin Pulm Med 2005; 4: 205-9.

[10] McShane PJ, Naureckas ET, Tino G, Strek ME. Non-cystic fibrosis bronchiectasis. Am J Respir Crit Care Med 2013; 188(6): 647-56.

[11] Saleh AD, Hurst JR. How to assess the severity of bronchiectasis. Eur Respir J 2014; 43: 1217-9.

[12] Martinez-Garcia MA, de Gracia J, Relat MV, et al. Multidimensional approach to non-cystic fibrosis bronchiectasis: the FACED score. Eur Respir J 2014; 43: 1357-67.

[13] Chalmers JD, Goeminne P, Aliberti S, et al. The Bronchiectasis Severity Index: an international derivation and validation study. Am J Respir Crit Care Med 2014; 189(5): 576-85.

[14] Brody AS, Klein JS, Molina PL, Quan J, Bean JA, Wilmott RW. High-resolution computed tomography in young patients with cystic fibrosis: distribution of abnormalities and correlation with pulmonary function tests. J Pediatr 2004; 145: 32-8.

[15] McGuinnes G, Naidich DP. CT of airway disease and bronchiectasis. Radiol Clin North Am 2002; 40: 1-19.

[16] Pasteur MC, Bilton D, Hill AT; British Thoracic Society Bronchiectasis non-CF Guideline Group. British Thoracic Society guideline for non-CF bronchiectasis. Thorax 2010; 65: i1-i58. 
[17] World Health Organization. Guidelines for controlling and monitoring the tobbaco epidemic. Geneva: WHO 1998.

[18] Smoking Pack-Years. Available from: http://smokingpackyears.cm/ calculate. [Accessed: 09.10.2104].

[19] Stenton C. The MRC breathlessness scale. Occup Med 2008; 58 : 226-7.

[20] Spirometry Guide: 2010 Update. Available from: http://www.goldc opd.org/uploads/users/files/GOLD_Spirometry_2010.pdf [Accessed 09.10.2014].

[21] Miller MR, Hankinson J, Brusasco V, et al. Standardisation of spirometry. Eur Respir J 2005; 26: 319-38.

[22] Pasteur MC, Hellwell SM, Houghton SJ, et al. An investigation into causative factors in patients with bronchiectasis: a prospective study. Am J Respir Crit Care Med 2000; 162: 1277-84.

[23] Naidich DP, McCauley DI, Khouri NF, Stitik FP, Siegelman SS. Computed tomography of bronchiectasis. J Comput Assist Tomogr 1982; 6: 437-44.
[24] Barker AF. Bronchiectasis. N Engl J Med 2002; 346: 1383-93.

[25] Fuschillo S, De Felice A, Balzano G. Mucosal inflammation in idiopathic bronchiectasis: cellular and molecular mechanisms. Eur Respir J 2008; 31: 396-406.

[26] King PT, Holdsworth SR, Farmer MW, Freezer N, Villanueva E, Holmes $P$. Characterization of the onset and presenting clinical features of adult bronchiectasis. Respir Med 2006; 100: 2183-9.

[27] De Souza A, Brown JS, Loebringer MR. Bronchiectasis Research \& Academic Network. Research priorities in bronchiectasis. Thorax 2013; 68: 695-6.

[28] Lange P, Marott JL, Vestbo J, et al. Prediction of the clinical course of chronic obstructive pulmonary disease using the new GOLD classification: a study of the general population. Am J Respir Crit Care Med 2012; 186: 975-81.

(C) Minov et al.; Licensee Bentham Open.

This is an open access article licensed under the terms of the Creative Commons Attribution Non-Commercial License (http://creativecommons.org/licenses/by-nc/3.0/) which permits unrestricted, non-commercial use, distribution and reproduction in any medium, provided the work is properly cited. 